shown to natively internalize such modified DNA. The basic parameters for this DNA probe internalization by the murine Krebs-2 tumor cells were characterized. Upon internalization, the fragments of DNA undergo partial and nonuniform hydrolysis of $3^{\prime}$ ends followed by ligation into a ring. The degree of hydrolysis, assessed by sequencing several clones with the insertion of specific PCR product, was 30-60 nucleotides. Conclusions: Results of the current investigation suggest the possibility to use Fap$\mathrm{dC}$-modified DNA fragments for further analysis of both membrane-associated and intracellular factors mediating the internalization of eDNA by Krebs-2 cells. Capability of Fap-dCDNA to impair the NER process presumes their possible applicability in antitumor therapy.

This study was supported by the Russian Science Foundation (project №17-74-10086).

References: 1. Evdokimov et al, DNA Repair 2018. 61: 86-98. 2. Dolgova et al., Cancer Biol Ther. 2014. 15: 1378-94. 3. Potter et al., Oncotarget. 2016. 10: 11580-94.

doi: http://dx.doi.org/10.7124/bc.0009B9

\section{A CRISPR view of the genome in living cells: cell cycle and genomic distance dependent dynamics of chromosomal loci}

Hanhui $\mathrm{Ma}^{1,5}$, Li-Chun $\mathrm{Tu}^{2}$, Yu-Chieh Chung $^{3}$, Ardalan Naseri ${ }^{4}$, David Grunwald ${ }^{2}$, Shaojie Zhang ${ }^{4}$, and Thoru Pederson ${ }^{1}$

${ }^{1}$ Department of Biochemistry and Molecular Pharmacology, University of Massachusetts Medical School, Worcester, MA 01605, USA; ${ }^{2}$ RNA Therapeutics Institute, University of Massachusetts
Medical School, Worcester, MA 01605, USA;

${ }^{3}$ Kavli Institute for the Physics and Mathematics of the Universe, University of Tokyo, Kashiwa, Chiba, 277-8583, Japan; ${ }^{4}$ Department of Computer Science, University of Central Florida, Orlando, FL 32816, USA; ${ }^{5}$ Present address: School of Life Science and Technology, ShanghaiTech University, Shanghai, China

thoru.pederson@umassmed.edu

In contrast to the well-studied condensation and folding of chromosomes during mitosis, their dynamics during interphase are less understood. We deployed our newly developed, brightnessenhanced CRISPR-based DNA imaging system (CRISPR-Sirius, Ma et al., 2018) to track the dynamics of genomic loci situated kilobases to megabases apart on a single chromosome. Two distinct modes of dynamics were resolved: local movements as well as translational movements of the entire domain within the nucleoplasm. The magnitude of both of these modes of movements increased from early to late G1, whereas the translational movements were reduced in early S. The local fluctuations decreased slightly in early $\mathrm{S}$ and more markedly in mid-late S. These results (Ma el al. J. Cell Biol., in press) suggest an ongoing compaction-relaxation dynamic of the interphase chromosome fiber, operating concurrently with changes in the extent of overall translational movements of loci in the 4D nucleome. The former possibility was anticipated some time ago (Pederson, 1972) and the latter shortly thereafter (Crick, 1978). It is too soon to know the full meaning of these interphase chromosome dynamics but all the tools to access this dimension of genome biology are fortunately now at hand.

Supported by NIH grants K99 GM126810 (L.-C.T), R01 GM102515 (S.Z.), U01 EB021238 (D.G.) and U01 
DA040588 (T.P.). The latter two grants are part of the 4D Nucleome Initiative of the NIH Common Fund.

References: Crick, F.H.C. 1978. Chromosome structure and function. Future prospects. Eur. J. Biochem. 83: 1-3. Ma, H., Tu, L.-C., Naseri, A., Chung, Y.C., Grunwald, D., Zhang, S. and Pederson, T. 2018. Engineered CRISPR RNA scaffolds with enhanced stability for signal amplification in genome imaging. Nature Methods 15: 928-931. Mazia, D. 1987. The chromosome cycle and the centrosome cycle in the mitotic cycle. Intl. Rev. Cytol. 100: 49-92. Pederson, T. 1972. Chromatin structure and the cell cycle. Proc. Natl. Acad. Sci. USA 69: 2224-2228.

doi: http://dx.doi.org/10.7124/bc.0009BA

\section{Poly(ADP-ribose) polymerases in regulation of DNA repair}

\section{Olga Lavrik}

Institute of Chemical Biology and Fundamental Medicine, Novosibirsk State University, Novosibirsk, Russia

The phenomenon of nicotinamide adenine dinucleotide (NAD+)-dependent poly(ADPribosyl)ation catalyzed with PARP1 was discovered long time ago, but it is still unclear how this post-translational modification governs a multitude of cellular processes including DNA repair. When interacting with the damaged DNA, PARP1 catalyzes the synthesis of a long branched poly (ADP-ribose) polymer (PAR) by using NAD + as a substrate. PAR can be attached to the acceptor amino acid residues of nuclear proteins or to PARP1 itself. This process leads to reorganization of the functional protein complexes involved in DNA repair. The aims of the present study were to investigate the role of poly (ADP-ribosyl)ation in regulation of base excision repair (BER), to search new targets of PARylation catalyzed with PARP1 and PARP2 and to analyze the modulation of PARPs activity by RNA binding proteins. Methods: Biochemical approaches, fluorescence titration methods, atomic force microscopy (AFM), light-scattering technique. Results: PARP1 interacts with BER proteins as well as with DNA intermediates of BER containing breaks or apurinic/apyrimidinic sites [1]. PARP1 and PARP2 activities regulate BER. The PARP1 activity is modulated by disordered RNA binding proteins such as YB-1 and FUS. YB-1 stimulates PARP1 activity [2]. The PARP-1/PAR/DNA system was reconstituted in vitro and the role of FUS was analyzed at the single molecule level [3]. The dissociation of FUS from mRNA, its recruitment at DNA damage sites through its binding to PAR, and the assembly of damaged DNA-rich compartments were demonstrated. PARG, an enzyme family that hydrolyses PAR, is sufficient to dissociate damaged DNA-rich compartments and PARG hydrolysis initiates the nucleocytoplasmic shuttling of FUS in cells. We anticipate that FUS facilitates a rapid DNA repair through the transient compartmentalization of DNA damages by FUS interaction with PAR. Conclusion: The results obtained show the key role of PARP1 in regulation of BER and the modulation of PARP1 activity by RNA binding proteins YB-1 and FUS.

This work was supported by grant from RSF 19-14-00107 (study of PARPs activity modulation by RNA binding proteins) and by Russian State funded budget project of ICBFM SB RAS (AAAA-A17-117020210022-4).

References: 1. Moor NA, Lavrik OI. Protein-Protein Interactions in DNA Base Excision Repair. Biochemistry (Mosc) 2018, 83:411-422. 2. Alemasova EE, Naumenko KN, Kurgina TA, Anarbaev RO, Lavrik OI. The multifunctional protein YB-1 potentiates PARP1 activity and decreases the efficiency of PARP1 inhibitors. Oncotarget. 2018, 9:23349-23365. 3. Singatulina AS, Hamon L, 